\title{
A COMBINATORIAL METHOD FOR HEALTH-CARE DISTRICTING
}

$$
\text { C.Ghiggi P.P.Puliafito R.Zoppoli }
$$

\author{
Istituto di Elettrotecnica \\ Universitè di Genova \\ Viale Causa, 13 \\ 16145 Genoa (Italy)
}

\section{INTRODUCTION}

The problem described in this paper arises whenever, for political or administrative reasons, a geographical region must be partitioned into an unknown number of districts so that, within each district, the total amount of service supplied by existing facilities of known location and capacity may satisfy a given demand, according to some optimality criterion.

The philosophy of the problem is quite different from that of the so-called. Iocation Problem (see for example TORTGAS et AI. [1]), since we are neither seeking locations for new facilities nor trying to modify their capacities.

The following assumptions will define the problem more clearly. 1. The region is considered to be made up of a certain number of assigned undivisible communities of known concentrated population.

2. Each community must be univocally assigned to a district, i.e., no overlap among districts is allowed.

3. The location and capacity of every facility are assigned.

4. Each district must be connected in the geometrical sense.

5. The population of each district must lie within an assigned range.

6. The number of districts is unknown.

In the following, we shall refer to hospitals as facilities.

This work was supported by the National Council of Research of Italy (CNR). 
The capacity of a facility will be simply characterized by the number of beds of the hospital. Then, the aim of the paper will be to present a partioning method for the region, such that each district can be considered as "self-sufficient" from a health care point of view, and "satisfactory" for both the planner and the citizen. Such terms will be specified later on.

It is worth noting that our problem formulation is comprehensive enough as to describe conveniently districting problems for other classes of facilities.

\section{STATEMENT OF THE PROBIEN}

Although analytical constraints may be derived from the preceding assumptions, reduction of our districting problem to a mathematical program does not seem an easy matter. Actually, as frequently happens in planning problems in which socio-economical factors are involved, it is rather unrealistic to define a single cost function that satisfactorily describes the several reguirements of a health care system, which cannot always be expressed in quantitative terms.

Since a formal approach to the problem based on the multigoal decision theory is beyond the aim of this paper, we will assume,for the time being, to have achieved a "satisfactory" cost function to be minimized. Then, it will be difficult to avoid what is commonly called an "ill-structured" formulation of the problem. This cost function should include such factors as the average time (or distance) travelled by the citizen to reach the hospital he is assigned to, and the difference between the demand and the offer of bed-units within the district. Other factors might have to be minimized such as, for instance, the number of districts (in order to reduce admin istrative expenses) or the difference between a given district's population and a pre-assigned value suggested by social or administrative reasons.

To state the structure of the problem more clearly, let us consider the geographical region shown in Fig.1. This region is 
subdivided into communities, a limited number of which have a hospi tal on their territory. Since the only information we need to retain from the map of Fig. 1 is adjacency between communities, we will refer to the graph of Fig.2, in which nodes correspond to communities and an arc between two nodes denotes an adjacency relationship between the corresponding communities. Obviously, according to the presence of a hospital in a community, two classes of nodes (with and without a hospital) can be aistinguished.
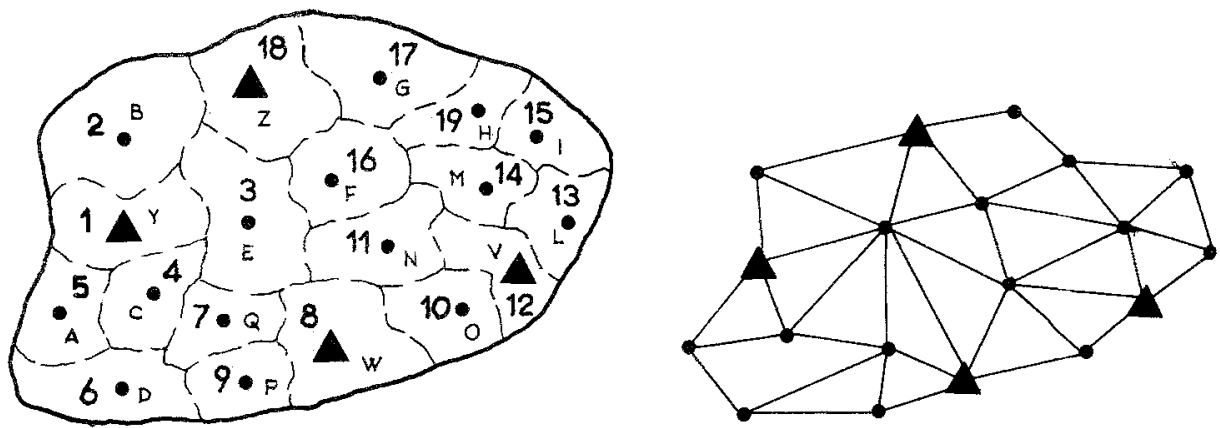

A Communities with Mospital

- Communities without Hospital

Fig. 1. Geographical region

Fig. 2. Graph of adjacent communities

Observe now that there is a one-to-one correspondence between the set of all trees in the graph and the set of all connected districts in the map. It follows that a tree including at least one hospital and characterized by an allowed amount of population yielos a "feasible district" in the sense of the assumptions introduced in Section I. These properties will lead to a computational procedure for finding all possible districts.

Let $Q \triangleq\left\{a_{1}, a_{2}, \ldots, a_{n}\right\}$ be the set of all nodes in the graph, and $u \triangleq\left\{u_{1}, u_{2}, \ldots, u_{m}\right\}$ the set of all feasible districts. Then, we define, as a "feasible solution" of the districting problem, every 
subset

$s_{i} \triangleq\left\{u_{i_{1}}, u_{i_{2}}, \ldots, u_{i_{k}}\right\} \in u$ such that

$$
\begin{aligned}
& u_{i_{1}} \cup u_{i_{2}} \cup \ldots \cup u_{i_{k}}=\varnothing \\
& u_{i_{j}} \cap u_{i_{h}}=\varnothing, \quad \forall j \neq h .
\end{aligned}
$$

Every feasible solution $s_{i}$ partitions the given region into nonoverlapping and connected districts of allowed population. Fig. 3 shows an example of feasible solution. We will assume that the set of feasible solutions is not an empty set.

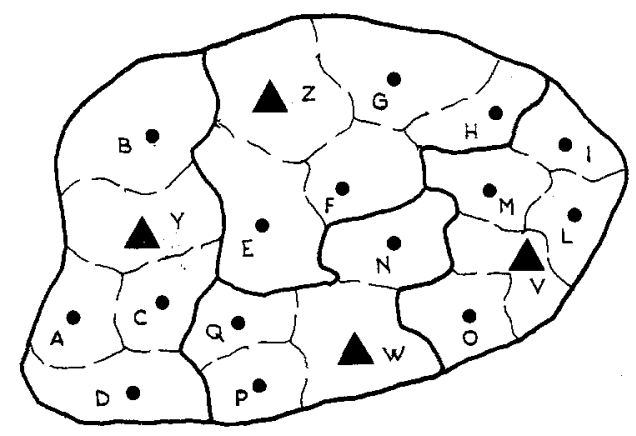

Fig. 3. Example of feasible solution.

From the above definitions, an algebraic formulation of the districting problem directly follows. Let us define an $\mathrm{n} x \mathrm{~m}$ constraint matrix $A=\left[a_{i j}\right]$ in the following way:

- every community (i.e., every node of $(0)$ corresponds to a row in $\mathrm{A}$;

- every feasible district (i.e., every element of $U$ ) corresponds to a column of $\mathrm{A}$;

$-a_{i j}=1$ if district $j$ includes community $i, a_{i j}=0$ otherwise. Any collection of feasible districts is then represented by a zero-one vector $\underline{x}=\left(x_{1}, x_{2}, \ldots, x_{m}\right)$, where $x_{j}=1$ if the collection 
includes district $i, x_{i}=0$ otherwise. Then, a vector $x$ will represent a feasible solution provided that the following constraints are verified:

$$
A \underline{x}=1
$$

where 1 is an n-vector of all 1s. Constraints (3) are the algebraic version of conditions (1), (2).

Assume now that, whatever optimality criterion may have been chosen for the districting problem, it is meaningful to characterize a given feasible district $i$ by means of a cost $c_{i}$, and this independentiy of the other districts selected for the feasible solution. Assume also that it is possible to "evaluate" any feasible solution by considering the total cost, which is obtained by adding the costs of all districts included in the solution.

Such "independence" and "additivity" assumptions enable us to define as "optimal solution" the feasible solution $\underline{x}^{*}$ that mini mizes the cost function

$$
z=\sum_{i=1}^{m} c_{i} x_{i}=\underline{c}^{\prime} \underline{x}
$$

where $\underline{c}=\left(c_{1}, c_{2}, \ldots, c_{m}\right)$. Cost (4) and constraints (3) give to our districting problem the more familiar form of a set Partitioning Problem (see, for example, ARONOFSKY [2]), which is a special tyoe in the class of integer programming problems. The solution of such a mathematical program will be discussed later on.

\section{A STMPLIFIED VERSTON OF THE PROBLEM}

Before we describe a method for finding all feasible districts (and thus for building the constraint matrix $A$ ) and an enumerative method for solving the Set Partitioning Problem, we must admit that the problem, as it is stated, is really usually too big for our means of solution. Then, some way must be found in order to reduce the dimensionality of the problem without wasting too much of its meaningfulness. In a typical region of Italy, the number of commu 
nities might easily be 400 or 500 , and the number of feasible districts, for a reasonable choice of the lower and upper population bounds, will inevitably grow to several thousands of units.

One way of facing this shortcoming might consiste in discarding many feasible districts on the basis of some intuitive judgement, for instance by not allowing, for particular geographical conditions, long and narrow or starred districts. Obviously, such simplification leads to reduce the number of columns of $A$ or, equivalently, the number of elements of the set $U$. As a result of this reduction, suboptimal solution (if they exist) will in general be found.

To avoid performing such a reduction too arbitrarily, a twolevel computational procedure is proposed.

1) At the first level, we reduce the set $U$ to a new set $\tilde{U}$ such that $\tilde{U} \subset U$ (then, a reduced matrix $\tilde{A}$ is obtained).

2) At the second level, we solve a simplified set Partitioning Prob lem on matrix $\widetilde{A}$, possibly after changing the cost vector $c$ to a new cost vector $\tilde{\tilde{c}}$, and obtain a suboptimal solution $\tilde{x}^{*}$ Let $\delta$ be the set of all feasible solutions $s_{i}$ that can be derived from $u$, and let $\tilde{\mathcal{S}}$ be the set that can be derived from $\tilde{U}$. clearly, $\tilde{\mathcal{S}} \subseteq \delta$. The introduction of the new cost vector $\tilde{\tilde{c}}$ would be unnecessary if one could know in advance that the optimal solution $\underline{x}^{*}$ still belongs to $\tilde{S}$.

To be more specific, let us examine in some detail how this procedure can work in the problem we are dealing with. As we said in Section II, the global cost function should include a term, penalizing the average time (or distance) travelled by the citizen to reach the hospital he is assigned to. If by exploiting the particular structure of the cost function, we first try to minimize such a term, it is easy to show that a meaningful reduction of set $U$ can be obtained, which is consistent with the intuition and simplifies the original set Partitioning Problem by several orders of magnitude.

What we essentially want to obtain at the first level is a clustering of the communties around the most appropriate hospitals 
so that the average distance is minimized with the constraint that the demand of bed-units is satisfied within every cluster. A natural and straightforward way to determine such clusters consists in solv ing a suitable Transportation Problem (see for esample HADLEY [3]) as follows.

Let us consider the inhabitants of the region as goods to be shipped from the $n$ communities to the 1 hospitals. Then, a Transportation Problem can be stated for which the cost function is given by

$$
t=\sum_{i=1}^{n} \sum_{j=1}^{1} z_{i j} c_{i j}, z_{i j} \geq 0
$$

where $z_{i j}$ is the number of people assigned from community $i$ to hospital $j$, and $c_{i j}$ is the distance (or the travel time) between community $i$ and hospital $j$. If hospital $j$ is within community $i$, then $c_{i j}=0$. Obvious constraints are the following:

$$
\sum_{j=1}^{1} z_{i j}=a_{i}, \quad i=1,2, \ldots, n
$$

where $a_{i}$ is the number of inhabitants of community $i$.

If we do not impose any other constraint, a trivial optimal solution wholly assigns every community to the nearest hospital regardless of its capacity. Recall, however, that we have assumed the number of beds for each hospital to be given. Let $b_{j}$ be the number of beds of hospital j. Since bed-units and inhabitants are not homogeneous quantities, we need some comparison coefficient to meet the demand and the offer of the health-care system. Let $\alpha$ be such a coefficient, which specifies the number of inhabitants that can be assisted by the health services corresponding to one bed-unit. The balance between demand and offer is then expressed by the following constraints

$$
\sum_{i=1}^{n} z_{i j} \leq \alpha b_{j}, \quad j=1,2, \ldots, 1
$$


Clearly, since we have assumed that no new bed needs to be added, we must have

$$
\sum_{i=1}^{n} a_{i} \leq \alpha \sum_{j=1}^{1} b_{j}
$$

or, equivalently,

$$
\alpha \geq \bar{\alpha}=\sum_{i=1}^{n} a_{i} / \sum_{j=1}^{I} b_{j}
$$

The choice of $\alpha$ will be discussed later on. Suppose now to have solved the Transportation Problem outlined by (5), (6), (7) for a certain value of $\alpha$. According to this solution, some communities will have been assigned to a unique hospital, while the remaining ones will have been shared among two or more hospitals. However, the number of the latter class of communities, which we will call "unassigned communities", is smaller than the number of hospitals, since in a Transportation Problem the number of nonnegative $z_{i j}$ is $\leq$ n+1-1. Fig.4 shows a typical solution of the Transportation Problem.

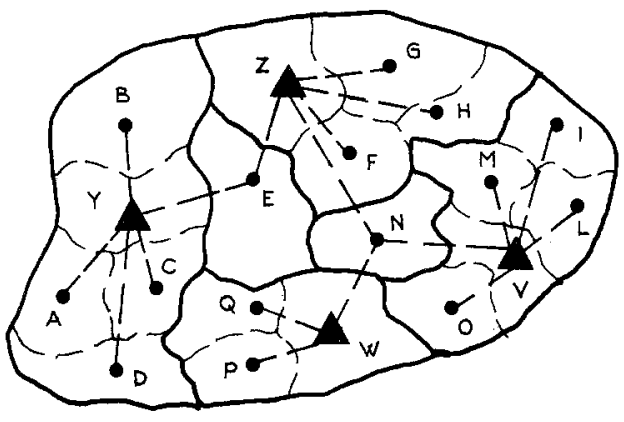

Fig.4. Solution of the Transportation Problem.

If the communities assigned to a hospital and the hospital itself constitute a connected subregion (and this happens most often), 
we define such subregion as a "kernel". It follows that the original graph of Fig.2 is reduced to a much simpler one, the nodes of which are given by kernels, unassigned communities and communities that have been assigned to a single hospital but that are not connected with the corresponding kernel (also these communities will be called "unassigned communities"). A reduced graph of this type is shown in Fig. 5.

The construction of the feasible districts, as illustrated in Section II, can now be performed on this graph, yielding the reduced set of feasible districts $\tilde{U}_{\alpha}$ (or,equivalently, the matrix $\tilde{A}_{\alpha}$ ) as required by the first computational level. The subscript $\alpha$ recalls that the reduced set $\widetilde{U}_{\alpha}$ and the matrix $\widetilde{A}_{\alpha}$ depend now on the factor $\alpha$.

At the second computational level, we must solve a new Set Partitioning Problem, which, however, is much simpler than the original one. Of course, the new cost vector $\tilde{\tilde{c}}$ will include other terme of the global optimality criterion, but not necessarily distance or travel time.

As regards the factor $\alpha$, we notice that this coefficient has, in our problem, both a mathematical and a socio-economical meaning. From a mathematical point of view, observe that the coefficient $\alpha$, in the range $(\bar{\alpha}, \infty)$, should be assigned a value such that $\underline{x}^{*} \in \widetilde{S}_{\alpha}$. Since such a choice is difficult to make beforehand (maybe a sensitivity analysis on $\alpha$ would be useful), a meaningful value may be selected on the basis of the planning re guirements. For instance, $\alpha$ might take on some standard value assigned by a national planning board, or take on the value $\bar{\alpha}$. In the latter case, in solving the Transportation Problem a uniform inhabitants / bed-units ratio would be assumed for the hospitals of the region.

In the next section, the scheme of an algorithm to pexform the two level computational procedure will be presented. 
IV. ALGORITHMS FOR SOLUTION

The first step towards the solution of our reduced Set Partitioning problem consists in finding an algorithm for building the constraint matrix $\tilde{\mathbb{A}}_{\alpha}$ column by column. Since the number of possi ble combinations with $I$ kernels and $K$ unassigned communities is $\left(2^{\mathrm{L}}-1\right) 2^{\mathrm{K}}$, to enumerate all such combinations and then to select all feasible districts may clearly turn out to be prohibitive.

Therefore, the search for the feasible districts will be tried by means of the following procedure:

1) choose any kernel as a root;

2) find on the reduced graph all trees spanning from this root and having population lying between the assigned limits;

3) choose another kernel as a new root, go back to step 2), and so on for all kernels.

The process of finding all trees spanning from an assigned root is readily accomplished by means of a search technique which is similar to the "Backtrack Programing" method proposed by GOIOMB [4]. The resultant algorithm backtracks over the solution stack every time the population exceeds the upper limit or a tree is outlined that has been found before.

Once the constraint matrix $\tilde{A}_{\alpha}$ is found, there exist several different approaches leading to the optimal solution $\underline{\underline{x}}^{*}$ (cutting planes, Balas algorithm, branch and bound, rounding off the solutions of a standard linear programming code, etc.; see also for references HU [5]). The one that seems to be the most appropriate for our problem is an implicit enumerative search technigue which, by means of a previous ordering of its columns, scans matrix $\tilde{\mathrm{A}}_{\alpha}$ looking for all feasible solutions.

This approach is due to PIERCE [6], who suggested a particular way of ordering the columns. Even though it may be slower than other methods, it has the great advantage of finding all feasible solutions. This might be the starting point for a multigoal opti mization or, more simply, it might allow the planner to assign dif 
ferent costs to every feasible solution, according to different objectives, and then to choose as "the optimal solution" the one that ranks most satisfactorily with respect to all objectives.

The rows of matrix $\widetilde{A}_{\alpha}$ are now given by the kernels and the unassigned communties, while the columns are the feasible districts; the elements of such a matrix have the same meaning as the elements of $A$ in (3).

The columns of matrix $\widetilde{\mathbb{A}}_{\alpha}$ are ordered in such a way that, in reading them from the left to the right, groups of columns are found in the following order: the columns of the first group have 1 in the first row, the columns of the second sroup have 0,1 in the first and in the second row, respectively, and so on. The following example will clarify such a structure.

$$
\tilde{A}_{\alpha}=\left(\begin{array}{llll|lll|lll|ll}
1 & 1 & 1 & 1 & 0 & 0 & 0 & 0 & 0 & 0 & 0 & 0 \\
1 & 0 & 0 & 0 & 1 & 1 & 1 & 0 & 0 & 0 & 0 & 0 \\
0 & 0 & 1 & 0 & 0 & 0 & 0 & 1 & 1 & 1 & 0 & 0 \\
0 & 1 & 0 & 0 & 1 & 0 & 0 & 1 & 0 & 0 & 1 & 1 \\
0 & 0 & 0 & 0 & 0 & 1 & 1 & 0 & 0 & 1 & 0 & 1 \\
0 & 1 & 1 & 0 & 0 & 0 & 1 & 0 & 0 & 0 & 1 & 0
\end{array}\right)
$$

Recalling that a feasible solution is a collection of feasible districts such that each kernel, or unassigned community, belongs to one and only one district, we reckon that a feasible solution cannot have two columns covering the same kernel or unassigned community (i.e., having a 1 in the same row). Thus, for instance, two columns that appear in a feasible solution cannot belong to the same group.

The algorithm chooses one column from the first group, then one from the second, checking that they both do not cover the same row, and so on, and backtracks whenever a feasible solution may be found beginning with the current columns. FLOYD's method [7] has been used to implement the backtrack alforithm.

A realistic example about the province of Imperia, Italy, is presented with the intent of clarifying our approach. The province is made up of 67 communities and it is served by 7 non-specialistic 
hospitals.

Fig. 6 shows the output of the Transportation Problem when an $\alpha=\bar{\alpha}$ has been chosen. Two hospitals have disappeared from the map, since their capacity was so small that, according to parameter $\alpha$, they were not even big enough for their community. Thus, the two communities have been erased from the number of destinations and have been added to the number of origins (with a reduced population).

By choosing the population limits for the feasible districts $P_{\text {min }}=30.000$ and $P_{\text {max }}=100.000$, the algorithm has generated the sixteen feasible solutions of Fig.7.

\section{CONCLUDING COMMENTS}

Two essential features characterize the method proposed in the paper: 1) the reduction of the original high-dimensional problem to a smaller one by solving a suitable Transportation Problem, 2) the choice of Backtrack Programming algorithms to face the subsequent combinatorial problems. Since the simplified approach of point one is heuristic and the choice of point two may appear to be arbitrary, some comments are needed.

As regards the first point, it is worth noting that, despite the heuristic nature of the suggested simplification, the introduc tion of the Transportation Problem to reduce the dimensionality of the original problem is quite consistent with the intuition, since it emphasizes the role of hospitals as gravitation centers for the health-care demand. Moreover, such a reduction may be "controlled" by means of a unigue parameter, i.e., the coefficient $\alpha$ which enables an easy sensitivity analysis significant for both a mathe matical and a socio-economical interpretation.

The choice of a Backtrack Programming approach to solve the final Set Partitioning Problem is probably a questionable fact, since other combinatorial methods may perform more efficiently. For the present problem other experimental work is required. 0 b 


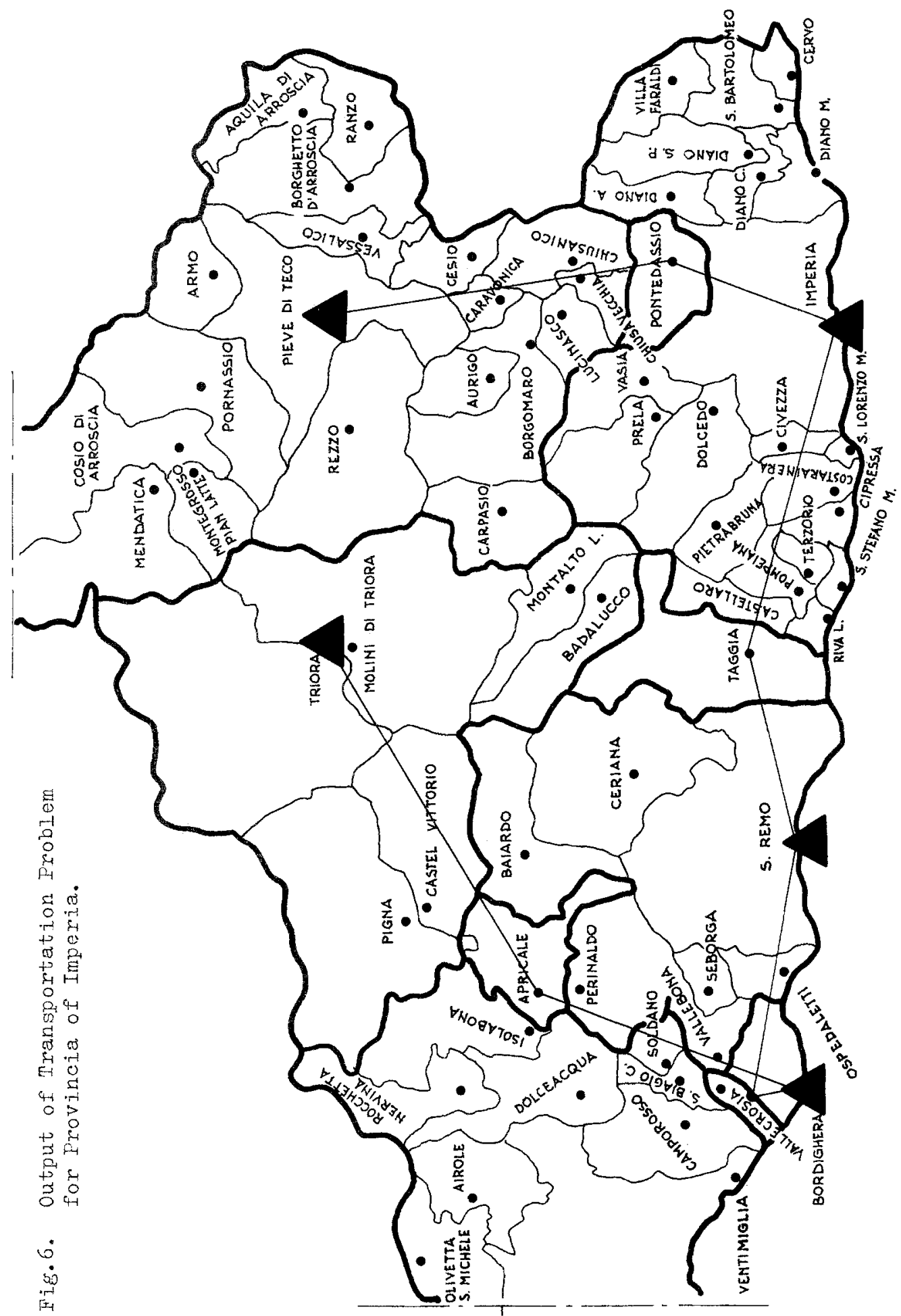



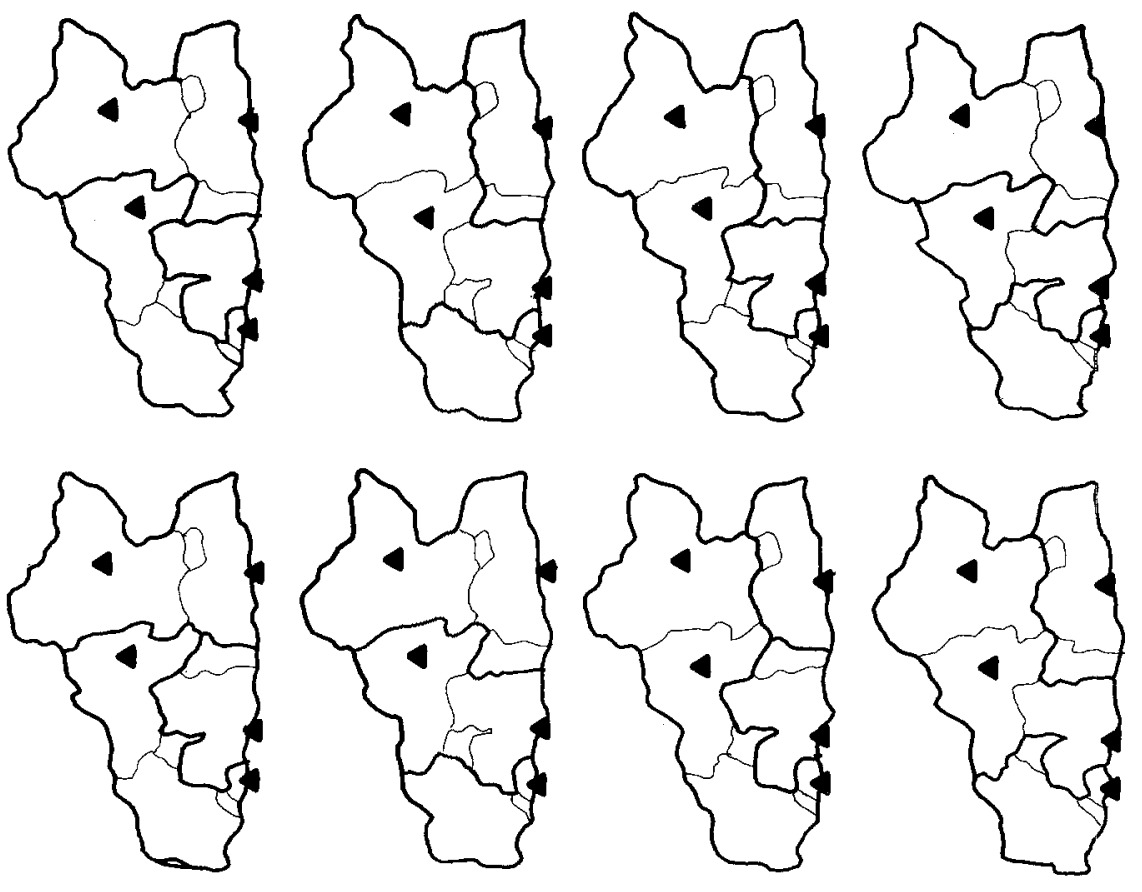

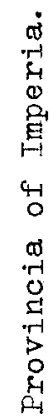
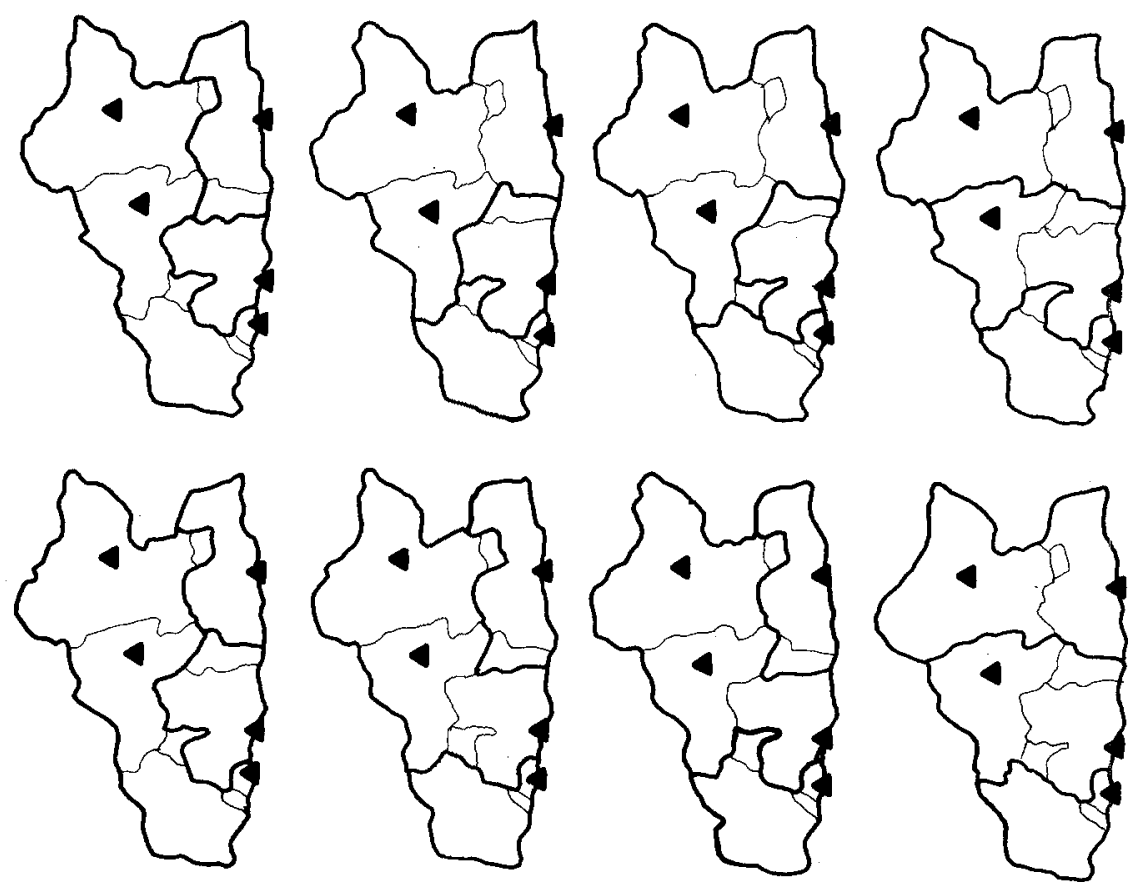
serve, however, that, for a given solution of the Transportation Problem, the search for feasible districts, or equivalently, the determination of the matrix $\widetilde{A}_{\alpha}$, is not at all an easy matter. For this search, no better tool has been found by the authors (for exsample, the computational time turns out to be approximately linear with the dimension of the region, although highly sensitive with respect to the upper population limit $\mathrm{P}_{\max }$ ). It is also interesting to observe that this kind of search may be facilitated, from a prac tical point of view, by using particular programming languages like, for instance, the Symmetric Iist Processor.

\section{REFERENCES}

[1] C.TOREGAS, R.SWAIN, C.REVELIE, I.BERGMAN: "The Location of Bmergency Service Facilities", Opns. Res, 19,1971, pp.1363-1373 .

[2] J.S.ARONOFSKY: "Progress in Operations Research", John Wiley and Son Inc., liew York, 1969.

[3] G.HADLEY: "Linear Programming", Addison-Wesley, Reading, Mass., 1962.

[4] S.W.GOLONB: "Backtrack Programming", JACM, 12,1965, pp.516-524 .

[5] T.C.HU: "Integer Frogramming and Network Flows", Addison-Wesley, Reading, Mass., 1970.

[6] J.F.PIERCE: "Application of combinatorial programming to a class of all zero-one Integer Programming problems", Rept. 36. Y03, IBM Data Processing Division, Cambriage Sc.Center.

[7] R.W.FLOYD: "Non Deterministic Algorithms", JACM, vol.14, n.4, oct.1967, pp.636-644. 